\title{
THE COMPUTATION OF RESONANCES IN OPEN SYSTEMS USING A PERFECTLY MATCHED LAYER
}

\author{
SEUNGIL KIM AND JOSEPH E. PASCIAK
}

\begin{abstract}
In this paper, we consider the problem of computing resonances in open systems. We first characterize resonances in terms of (improper) eigenfunctions of the Helmholtz operator on an unbounded domain. The perfectly matched layer (PML) technique has been successfully applied to the computation of scattering problems. We shall see that the application of PML converts the resonance problem to a standard eigenvalue problem (still on an infinite domain). This new eigenvalue problem involves an operator which resembles the original Helmholtz equation transformed by a complex shift in the coordinate system. Our goal will be to approximate the shifted operator first by replacing the infinite domain by a finite (computational) domain with a convenient boundary condition and second by applying finite elements on the computational domain. We shall prove that the first of these steps leads to eigenvalue convergence (to the desired resonance values) which is free from spurious computational eigenvalues provided that the size of computational domain is sufficiently large. The analysis of the second step is classical. Finally, we illustrate the behavior of the method applied to numerical experiments in one and two spatial dimensions.
\end{abstract}

\section{INTRODUCTION}

In this paper, we consider the approximation of resonance values in open systems using approximations coming from the PML (perfectly matched layer) technique. Problems involving resonances in open systems result from many applications, including the modeling of slat and flap noise from an airplane wing, gravitational waves in astrophysics, and quantum mechanical systems. We consider the approximation of resonances in the frequency domain in this paper.

As a model problem, we consider a resonance problem in three-dimensional space which results from a compactly supported perturbation of the Laplacian, i.e.,

$$
L u=-\Delta u+L_{1} u,
$$

where $L_{1}$ is symmetric and lives on a bounded domain $\Omega \subset \mathbb{R}^{3}$. We then seek $k$ such that there are non-trivial "eigenfunctions" $\psi$ satisfying

$$
L \psi=k^{2} \psi \text {. }
$$

Received by the editor July 9, 2007 and, in revised form, July 22, 2008.

2000 Mathematics Subject Classification. Primary 65N30, 78M10.

Key words and phrases. Perfectly matched layer, PML, resonances, nonsymmetric eigenvalue problem.

This work was supported in part by the National Science Foundation through grant DMS0609544. 
The function $\psi$ is required to satisfy an outgoing condition corresponding to the wave number $k$. A resonance value $k$ corresponds to an improper eigenvalue problem, and the corresponding eigenvector grows exponentially. This exponential growth makes the problem difficult to formulate and even more difficult to approximate numerically.

The PML technique was introduced by Bérenger for scattering problems [4, 5. The idea was to surround the area of computational interest by an absorbing media (layer) which was perfectly free of reflection. Bérenger's original formulation involved splitting the equations in the absorbing layer. Subsequent formulations avoid this splitting and can often be interpreted as a complex stretching; cf., [10]. A properly devised complex stretching (or change of variable) preserves the solution inside the layer while introducing exponential decay at infinity. Thus, it is natural to truncate the problem to a finite domain, introduce a convenient boundary condition on the artificial boundary and apply the finite element method. This is a very successful technique which has been investigated both theoretically and computationally.

For scattering problems, one traditionally introduces a PML stretching which depends on the wave number yielding wave number independent decay 21. In contrast, for PML resonance problems, we use a stretching independent of the wave number. This results in wave number dependent decay. This is important in that when this decay is stronger than the exponential growth of the resonance eigenfunction, this eigenfunction is transformed into a proper eigenfunction for the PML equation (still on the infinite domain). In fact, the resulting PML eigenfunction still has residue exponential decay. Because of this decay, it is natural to consider truncating to a finite domain and subsequently applying the finite element method. The goal of this paper is to study the eigenvalue behavior when the eigenvalues of the (infinite) PML problem are approximated by those corresponding to a finite element method on a truncated computational domain.

The application of PML for the computation of resonances is not new. It turns out that PML is related to what is called "spectral deformation theory", developed by Aguilar, Balslev, Combes and Simon [1, 3, 16, 24. This theory shows that the PML eigenvalues for the infinite domain problem coincide with the resonance values of the original problem for rather general scaling schemes. The experimental behavior of truncated PML approximations to resonances in open systems was investigated in [13, 14. Our paper provides the first theoretical analysis of the convergence of the truncated PML eigenvalue approximation.

In many applications, the convergence of approximate eigenvalues is the result of classical perturbation theory (cf. [17). Indeed, one is able to conclude basic eigenvalue convergence provided that one can show that approximate operators converge in norm to the appropriate continuous operator. The PML eigenvalue problem is interesting as norm convergence does not hold in this case, and so eigenvalue convergence has to be proved in a more basic way.

The convergence of the eigenvalues obtained by finite element discretization has had a long history of research. Osborn [23] and Bramble-Osborn [8] consider the case of second order elliptic problems on bounded domains. In this case, the solution operator is compact and it is possible to prove convergence in norm of the discrete approximation. More recent work has been done on the Maxwell eigenvalue problem [6, 7, 19, 22]. This problem is more difficult than the uniformly elliptic 
problem mentioned above due to a non-compact inverse. However, in this case, the only trouble is due to gradient fields and results in a non-discrete spectrum consisting of only one point, the origin. In contrast, the PML problem is posed on an infinite domain and its inverse has essential spectrum, while the inverse of the truncated PML problem is compact. Accordingly, there cannot be norm convergence and the analysis of the convergence of eigenvalues/eigenvector must proceed in a non-standard fashion. Of course, the critical property here is the decay of the approximated eigenfunctions, and it is this property which is central to our analysis.

We note that the convergence of the eigenvalues associated with the finite element approximation to those of the PML problem on the truncated domain is standard; see Bramble and Osborn [8. Their results can be used to show that the corresponding discrete eigenvalues converge to those of the continuous PML problem (on the truncated domain) without the occurrence of spurious modes. Thus, the goal of this paper is to analyze the convergence (as the size of the truncated domain increases) of the eigenvalues of the continuous truncated problem to those of the infinite PML problem.

The question of spurious eigenvalues is delicate. First of all, numerical methods at any level of discretization usually generate some numerical eigenvalues which have nothing to do with the actual eigenvalues. For example, high frequency eigenvector components in a Galerkin approximation to the Dirichlet problem on a bounded domain exhibit little eigenvalue accuracy. Fortunately, the corresponding eigenvalues remain far away from the lower eigenvalues which have the desired convergence. Thus, the goal is to have a method where the eigenvalues of interest are approximated well and can be easily identified from the extraneous numerical ones.

The PML resonance case is much more complicated. Computational eigenvalues not related to the resonance values result from the essential spectrum associated with the infinite domain PML problem, the domain truncation, the mesh size and the placement of the PML layer. At least in the model problem which we are considering, the location of the essential spectrum is a priori known, so the numerical eigenvalues associated with it are easy to identify. Other "spurious like" eigenvalues can be moved away by changing the layer placement, truncation domain size and mesh parameter. Our theorems guarantee convergence at the resonance values provided that the mesh parameter is sufficiently small and the computational domain is sufficiently large. How to choose all of these parameters in such a way as to guarantee that the resonances of interest in an actual application have been located is a difficult question.

The organization of the remainder of this paper is as follows. In Section 2, the PML method is applied to resonance problems resulting from a compactly supported perturbation of the Laplacian. There we show how PML turns the resonance problem into a standard eigenvalue problem. In Section 3, the truncated PML problem is introduced, and we show that any compact subset of the resolvent set for the infinite domain PML operator is contained in the resolvent set for the finite domain operator provided that the truncation domain is sufficiently large. This shows that there are no spurious eigenvalues associated with the domain truncation. In Section 4, we use the exponential decay of eigenfunctions for both the infinite and truncated PML problems to analyze the convergence of eigenvalues. Finally, in Section 5, the 
results of numerical experiments are provided which illustrate the behavior of PML approximations on some simple resonance problems.

\section{PML AND THE RESONANCE PROBLEM}

We consider a linear operator

$$
L=-\Delta+L_{1},
$$

where $L_{1}$ is a linear operator with support contained in the ball $\bar{\Omega}_{0}$ centered at the origin of radius $r_{0}$. For example, we can consider Schrödinger operators $-\Delta+V$ with a real valued potential $V$ supported in $\bar{\Omega}_{0}$. We shall concentrate on this example, as more general applications are similar.

We consider the Helmholtz problem:

$$
L u-k^{2} u=f \text { on } \mathbb{R}^{3} .
$$

Here $k$ is a complex number and the support of $f$ is contained in $\bar{\Omega}_{0}$. We need to set a "boundary condition" at infinity. We consider solutions which are outgoing. Since $L$ coincides with $-\Delta$ outside of $\Omega_{0}, u$ can be expanded in terms of spherical Hankel functions $h_{n}^{1}, h_{n}^{2}$ and spherical harmonics $Y_{n}^{m}$ (see, e.g., [12, 21] for details). We will define the outgoing solutions as the solutions with an expansion of the form

$$
u(x)=\sum_{n=0}^{\infty} \sum_{m=-n}^{n} a_{n, m} h_{n}^{1}(k r) Y_{n}^{m}(\hat{x}) \text { for } r=|x| \geq r_{0} .
$$

Here $\hat{x}=x /|x|$ and $a_{n, m}$ are constants. Resonances are solutions of (2.1) with $f=0$ satisfying the outgoing condition.

We shall be interested in weak solutions of (2.1) which are, at least, locally in $H^{1}$. This means that the series (2.2) converges in $H^{1 / 2}\left(\Gamma_{0}\right)$ where $\Gamma_{0}$ is the boundary of $\Omega_{0}$. It follows that the series converges in $H^{1}$ on any annular domain $r_{0}<r<R$ (see Theorem 2.2 below).

Remark 2.1. For $k$ real and positive, the outgoing condition coincides with the Sommerfeld condition

$$
\lim _{r \rightarrow \infty} r\left(\frac{\partial u}{\partial r}-i k u\right)=0 .
$$

For resonances, the resonance value $k$ has a negative imaginary part and the "eigenfunction" $u$ increases exponentially as $r$ becomes large. Such a $u$ does not satisfy (2.3). Note that the symmetry of $L$ prohibits the existence of exponentially decreasing eigenfunctions (which would satisfy (2.3)), with eigenvalues having the nonzero imaginary part. Thus any numerical method for computing resonances based on (2.3) is questionable at best.

The PML approach 9] provides a convenient way to deal with (2.1) with the outgoing condition. We will use a spherical PML (see Figure 1). Let $r_{1}$ be greater than $r_{0}$ and $\Omega_{1}$ denote the open ball of radius $r_{1}$ centered at the origin with the boundary $\Gamma_{1}$.

The PML problem is defined in terms of a function $\tilde{\sigma} \in C^{2}\left(\mathbb{R}^{+}\right)$satisfying

$$
\tilde{\sigma}(r)=\left\{\begin{array}{lcc}
0 & \text { for } & 0 \leq r<r_{0} \\
\text { increasing } & \text { for } & r_{0} \leq r<r_{1} \\
\sigma_{0} & \text { for } & r_{1} \leq r
\end{array}\right.
$$




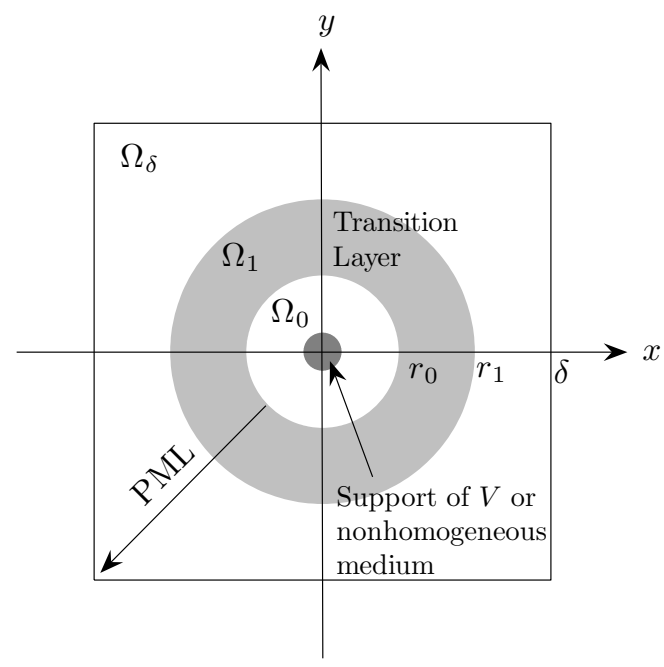

FiguRE 1. A cross-section of a spherical perfectly matched layer in $\mathbb{R}^{3}$

A typical $C^{2}$ function with this property is given in $\left[r_{0}, r_{1}\right]$ by

$$
\tilde{\sigma}(r)=\sigma_{0} \frac{\int_{r_{0}}^{r}\left(t-r_{0}\right)^{2}\left(r_{1}-t\right)^{2} d t}{\int_{r_{0}}^{r_{1}}\left(t-r_{0}\right)^{2}\left(r_{1}-t\right)^{2} d t} .
$$

The PML approximation can be thought of as a formal complex shift in coordinate system. We shall use the following notation:

$$
\begin{aligned}
\tilde{d}(r) & \equiv 1+i \tilde{\sigma}(r) \\
\tilde{r}(r) & \equiv r(1+i \tilde{\sigma}(r))=r \tilde{d} \\
\sigma(r) & \equiv \frac{\partial(r \tilde{\sigma})}{\partial r}=\tilde{\sigma}(r)+r \frac{\partial \tilde{\sigma}(r)}{\partial r}, \\
d(r) & \equiv \frac{\partial(\tilde{r}(r))}{\partial r}=1+i \sigma(r), \\
d_{0} & \equiv 1+i \sigma_{0}=\tilde{d}(r)=d(r) \text { for } r \geq r_{1} .
\end{aligned}
$$

The PML solution is defined by

$$
\tilde{u}(x)=\left\{\begin{array}{l}
u(x) \text { for }|x| \leq r_{0}, \\
\sum_{n=0}^{\infty} \sum_{m=-n}^{n} a_{n, m} h_{n}^{1}(k \tilde{r}) Y_{n}^{m}(\hat{x}) \text { for } r=|x| \geq r_{0} .
\end{array}\right.
$$

Clearly, $\tilde{u}$ and $u$ coincide for $|x| \leq r_{0}$. Moreover, $\tilde{u}$ satisfies

$$
\widetilde{L} \tilde{u}-k^{2} \tilde{u}=f \text { in } \mathbb{R}^{3},
$$


where $\widetilde{L}$ coincides with $L$ for $|x| \leq r_{0}$ and is given, in spherical coordinates $(r, \theta, \phi)$, by

$$
\begin{gathered}
\widetilde{L} v=-\left(\frac{1}{\tilde{d}^{2} d r^{2}} \frac{\partial}{\partial r}\left(\frac{\tilde{d}^{2} r^{2}}{d} \frac{\partial v}{\partial r}\right)+\frac{1}{\tilde{d}^{2} r^{2} \sin \theta} \frac{\partial}{\partial \theta}\left(\sin \theta \frac{\partial v}{\partial \theta}\right)\right. \\
\left.+\frac{1}{\tilde{d}^{2} r^{2} \sin ^{2} \theta} \frac{\partial^{2} v}{\partial \phi^{2}}\right)+V v
\end{gathered}
$$

We shall see that (2.5) has a well posed variational formulation in $H^{1}\left(\mathbb{R}^{3}\right)$ when $k$ is real and positive. Let $\chi$ be in $C_{0}^{\infty}\left(\mathbb{R}^{3}\right)$. Assuming that $\tilde{u}$ is locally in $H^{1}\left(\mathbb{R}^{3}\right)$, we have

$$
A(\tilde{u}, \chi)-k^{2} B(\tilde{u}, \chi)=\left(\tilde{d}^{2} f, \chi\right)_{\mathbb{R}^{3}}
$$

where

$$
\begin{aligned}
A(\tilde{u}, \chi) \equiv & \left(\frac{\tilde{d}^{2}}{d} \frac{\partial \tilde{u}}{\partial r}, \frac{\partial}{\partial r}\left(\frac{\chi}{\bar{d}}\right)\right)_{\mathbb{R}^{3}}+\left(\frac{1}{r^{2}} \frac{\partial \tilde{u}}{\partial \theta}, \frac{\partial \chi}{\partial \theta}\right)_{\mathbb{R}^{3}} \\
& +\left(\frac{1}{r^{2} \sin ^{2} \theta} \frac{\partial \tilde{u}}{\partial \phi}, \frac{\partial \chi}{\partial \phi}\right)_{\mathbb{R}^{3}}+(V \tilde{u}, \chi)_{\Omega_{0}}
\end{aligned}
$$

and

$$
B(\tilde{u}, \chi) \equiv\left(\tilde{d}^{2} \tilde{u}, \chi\right)_{\mathbb{R}^{3}} .
$$

To obtain a uniqueness result for the PML problem for $k$ real and positive, we introduce the following theorem. Let $A_{r_{0}, r_{2}}$ be an annulus bounded by two spheres of radius $r_{0}<r_{2}$.

Theorem 2.2. Let $k$ be a non-zero complex number not on the negative real axis. Suppose that $u \in H^{1}\left(A_{r_{0}, r_{2}}\right)$ and satisfies $A(u, v)=k^{2} B(u, v)$ for all $v \in C_{0}^{\infty}\left(A_{r_{0}, r_{2}}\right)$; then

$$
u(x)=\sum_{n=0}^{\infty} \sum_{m=-n}^{n}\left(a_{n, m} h_{n}^{1}(k \tilde{r})+b_{n, m} h_{n}^{2}(k \tilde{r})\right) Y_{n}^{m}(\hat{x}),
$$

and the series converges in $H^{1}\left(A_{r_{0}, r_{2}}\right)$.

Sketch of the proof. For $u \in H^{1}\left(A_{r_{0}, r_{2}}\right)$, by the $L^{2}$-orthogonality of $\left\{Y_{n}^{m}\right\}, u$ can be written as

$$
u(x)=\sum_{n=0}^{\infty} \sum_{m=-n}^{n} f_{n, m}(|x|) Y_{n}^{m}(\hat{x}) .
$$

The series above converges in the $L^{2}$ sense on each sphere $|x|=r$ with $r_{0} \leq r \leq r_{1}$ and also in $L^{2}\left(A_{r_{0}, r_{2}}\right)$. First, one shows that $f_{n, m}$ is in $H^{2}\left(\left[r_{0}, r_{2}\right]\right)$ and satisfies

$$
\frac{1}{r^{2} \tilde{d}^{2} d} \frac{\partial}{\partial r}\left(\frac{r^{2} \tilde{d}^{2}}{d} \frac{\partial f_{n, m}(r)}{\partial r}\right)+\left(k^{2}-\frac{n(n+1)}{r^{2} \tilde{d}^{2}}\right) f_{n, m}(r)=0 .
$$

Next, one shows that the initial value problem (2.12) with the initial conditions $f_{n, m}\left(r_{0}\right)$ and $f_{n, m}^{\prime}\left(r_{0}\right)$ given has a unique solution in $H^{2}\left(\left[r_{0}, r_{2}\right]\right)$. Since $h_{n}^{1}(k \tilde{r})$ and $h_{n}^{2}(k \tilde{r})$ solve (2.12), it follows that

$$
f_{n, m}(r)=a_{n, m} h_{n}^{1}(k \tilde{r})+b_{n, m} h_{n}^{2}(k \tilde{r})
$$

for suitable constants $a_{n, m}$ and $b_{n, m}$. 
Using the $L^{2}$-orthogonality of $\left\{Y_{n}^{m}\right\}$, it is not hard to see that a function $g \in$ $L^{2}\left(\Gamma_{j}\right)$ is in $H^{1}\left(\Gamma_{j}\right)$ for $j=0,2$ if and only if the series

$$
\sum_{n, m}(1+n(n+1))\left|\left(g, Y_{n}^{m}\right)_{\Gamma_{j}}\right|^{2} \equiv\|g\|_{H^{1}\left(\Gamma_{j}\right)}^{2}
$$

is finite. By interpolation, $g$ is in $H^{1 / 2}\left(\Gamma_{j}\right)$ if and only if the series

$$
\sum_{n, m}(1+n(n+1))^{1 / 2}\left|\left(g, Y_{n}^{m}\right)_{\Gamma_{j}}\right|^{2} \equiv\|g\|_{H^{1 / 2}\left(\Gamma_{j}\right)}^{2}
$$

is finite. This shows that the series (2.11) at $r_{0}$ and $r_{2}$ converge in $H^{1 / 2}\left(\Gamma_{0}\right)$ and $H^{1 / 2}\left(\Gamma_{2}\right)$ respectively. Let $\tilde{u}$ denote a partial sum in the series (2.10) and $\tilde{g}_{j}$ denote its trace to $\Gamma_{j}$ for $j=0,2$. We note that $\tilde{u} \in H^{1}\left(A_{r_{0}, r_{2}}\right)$ satisfies the variational problem,

$$
\begin{aligned}
A(\tilde{u}, \bar{d} \phi) & =k^{2} B(\tilde{u}, \bar{d} \phi) \text { for all } \phi \in H_{0}^{1}\left(A_{r_{0}, r_{2}}\right), \\
\tilde{u} & =\tilde{g}_{0} \text { on } \Gamma_{0}, \\
\tilde{u} & =\tilde{g}_{2} \text { on } \Gamma_{2} .
\end{aligned}
$$

Examining the coefficients appearing in the form on the left hand side above, we see that this is a well posed variational problem since the real parts of $\tilde{d}^{2} / d$ and $d$ are positive and uniformly (as $r$ varies) bounded away from zero. It follows that

$$
\|\tilde{u}\|_{H^{1}\left(A_{r_{0}, r_{2}}\right)} \leq C\left(\|\tilde{u}\|_{L^{2}\left(A_{r_{0}, r_{2}}\right)}+\left\|\tilde{g}_{0}\right\|_{H^{1 / 2}\left(\Gamma_{0}\right)}+\left\|\tilde{g}_{2}\right\|_{H^{1 / 2}\left(\Gamma_{2}\right)}\right),
$$

which implies convergence of (2.10) in $H^{1}\left(A_{r_{0}, r_{2}}\right)$.

The PML problem corresponding to a scattering problem was studied in [9], and the techniques there easily extend to our problem. The uniqueness of solutions to the PML problem (2.7) (for $k$ real and positive) now follows from the above theorem and the proof of Theorem 1 of [1]. Following [9], the perturbed form

$\widetilde{A}(u, \chi)=\left(\frac{\tilde{d}^{2}}{d^{2}} \frac{\partial u}{\partial r}, \frac{\partial \chi}{\partial r}\right)_{\mathbb{R}^{3}}+\left(\frac{1}{r^{2}} \frac{\partial u}{\partial \theta}, \frac{\partial \chi}{\partial \theta}\right)_{\mathbb{R}^{3}}+\left(\frac{1}{r^{2} \sin ^{2} \theta} \frac{\partial u}{\partial \phi}, \frac{\partial \chi}{\partial \phi}\right)_{\mathbb{R}^{3}}-d_{0}^{2} k^{2}(u, \chi)_{\mathbb{R}^{3}}$

is coercive. This follows by showing that the coefficients $\left\{\tilde{d}^{2} / d^{2}, 1,-d_{0}^{2}\right\}$ are all bounded away from zero and lie in a sector $-\pi+\epsilon \leq \arg (z) \leq 0$ for some positive $\epsilon$. As $A_{k}(w, v) \equiv A(w, v)-k^{2} B(w, v)$ is a low order perturbation of $\widetilde{A}(w, v)$ on a bounded domain, the inf-sup condition,

$$
\|u\|_{H^{1}\left(\mathbb{R}^{3}\right)} \leq C_{k} \sup _{\phi \in H^{1}\left(\mathbb{R}^{3}\right)} \frac{\left|A_{k}(u, \phi)\right|}{\|\phi\|_{H^{1}\left(\mathbb{R}^{3}\right)}} \text { for all } u \in H^{1}\left(\mathbb{R}^{3}\right),
$$

follows from uniqueness (see [9] for details). The analogous inf-sup condition for the adjoint operator holds as well:

$$
\|\phi\|_{H^{1}\left(\mathbb{R}^{3}\right)} \leq C_{k} \sup _{u \in H^{1}\left(\mathbb{R}^{3}\right)} \frac{\left|A_{k}(u, \phi)\right|}{\|u\|_{H^{1}\left(\mathbb{R}^{3}\right)}} \text { for all } \phi \in H^{1}\left(\mathbb{R}^{3}\right) .
$$

Remark 2.3. The inf-sup conditions above imply stability of the problem involving $A_{k}(\cdot, \cdot)$ on $H^{1}\left(\mathbb{R}^{3}\right)$. Part of the difficulty in extending the results developed in this paper to other types of PML is the derivation of analogous results. For example, elliptical PML [20] results in a PML equation which is non-constant at infinity. However, this problem seems tractable, as the coefficients become "almost" constant. Cartesian PML appears much more difficult, as the verification of stability in $H^{1}\left(\mathbb{R}^{3}\right)$ appears to require significantly different tools [18. 
Fix $k=1$ above. We define $T: L^{2}\left(\mathbb{R}^{3}\right) \rightarrow H^{1}\left(\mathbb{R}^{3}\right)$ by $T(f)=w$, where $w$ is the unique solution of

$$
A_{1}(w, \phi)=B(f, \phi) \quad \text { for all } \phi \in H^{1}\left(\mathbb{R}^{3}\right) .
$$

That $T$ is a bounded operator of $L^{2}\left(\mathbb{R}^{3}\right)$ into $H^{1}\left(\mathbb{R}^{3}\right)$ follows easily from (2.13) and (2.14). We can clearly restrict $T$ to an operator on $H^{1}\left(\mathbb{R}^{3}\right)$, and so its resolvent and spectrum are well defined.

Given a function $\tilde{u}$ satisfying (2.4), we can define $u(\tilde{u})$ by $u(\tilde{u})=\tilde{u}$ for $r \leq r_{0}$ and $u(\tilde{u})$ given by the series (2.2) for $r>r_{0}$ (with coefficients from the series for $\tilde{u})$.

The following theorem connects the resonance values with the eigenvalues of the PML operator $T$. This result for general stretching functions $\tilde{\sigma}$ is given by the Aguilar-Balslev-Combes-Simon Theorem [16]. However, the definitions required for their theory and its connection to the PML formulation above would require a lengthy discussion. Instead, we present a simple proof which works when the solutions of the PML problem are available in the explicit form (2.4).

Theorem 2.4. Let $\operatorname{Im}\left(d_{0} k\right)$ be greater than zero and set $\lambda=1 /\left(k^{2}-1\right)$. If there is a non-zero outgoing solution u (locally in $H^{1}$ ) satisfying (2.1) with $f=0$, then $\tilde{u}$ given by (2.4) is an eigenfunction for $T$ with an eigenvalue $\lambda$. Conversely, if $\tilde{u}$ is an eigenfunction for $T$ with an eigenvalue $\lambda$, then $\tilde{u}$ is of the form (2.4) for $r \geq r_{0}$ and $u=u(\tilde{u})$ satisfies (2.1) with $f=0$ and is outgoing.

For the proof of the above theorem, we shall require the following proposition.

Proposition 2.5. Let $\beta$ be a constant with positive imaginary part and $g$ be given in $H^{1 / 2}\left(\Gamma_{1}\right)$. There is a unique $w \in H^{1}\left(\Omega_{1}^{c}\right)$ satisfying $w=g$ on $\Gamma_{1}$ and

$$
\Delta w+\beta^{2} w=0 \text { on } \Omega_{1}^{c} .
$$

Moreover, $w$ is outgoing (the series representation given by Theorem 2.2 has vanishing $\left.b_{k}\right)$.

Proof. Consider the sesquilinear form

$$
a(u, v)=(\nabla u, \nabla v)_{\Omega_{1}^{c}}-\left(\beta^{2} u, v\right)_{\Omega_{1}^{c}}
$$

for $u, v \in H^{1}\left(\Omega_{1}^{c}\right)$. Since $\operatorname{Im}(a(u,-1 / \bar{\beta} u)) \geq C\|u\|_{H^{1}\left(\Omega_{1}^{c}\right)}^{2}$, it is straightforward to see that there is a unique $w$ in $H^{1}\left(\Omega_{1}^{c}\right)$ satisfying

$$
\begin{aligned}
a(w, v) & =0 \text { for all } v \in H_{0}^{1}\left(\Omega_{1}^{c}\right), \\
w & =g \text { on } \Gamma_{1} .
\end{aligned}
$$

Terms involving $b_{n, m}$ in the series of Theorem 2.2 blow up exponentially at infinity. The presence of any one results in a function not in $H^{1}\left(\Omega_{1}^{c}\right)$, i.e., $w$ is outgoing.

Remark 2.6. It follows from the above proposition and the proof of Theorem 2.2 that an outgoing series (with such $\beta$ ) which coincides with a function in $H^{1 / 2}\left(\Gamma_{1}\right)$, in fact, converges in $H^{1}\left(\Omega_{1}^{c}\right)$.

Proof of Theorem 2.4. Suppose that $u$ is outgoing, locally in $H^{1}$ and satisfies (2.1) with $f=0$. Then $u$ has a series representation (2.2). The resulting $\tilde{u}$ defined by (2.4) converges uniformly on compact sets of $\Omega_{0}^{c}$. It follows from the definition of $\widetilde{L}$ and the uniform convergence that $\tilde{u}$ satisfies

$$
\left(\widetilde{L}-k^{2}\right) \tilde{u}=0 .
$$


Outside of $\Omega_{1}$, this coincides with (2.15) with $\beta=d_{0} k$. Theorem 2.2 and Remark2.6 imply that the series for $\tilde{u}$ converges in $H^{1}\left(\Omega_{1}^{c}\right)$, i.e., $\tilde{u} \in H^{1}\left(\mathbb{R}^{3}\right)$. For $\phi \in C_{0}^{\infty}\left(\mathbb{R}^{3}\right)$,

$$
A(\tilde{u}, \phi)-k^{2} B(\tilde{u}, \phi)=0 .
$$

This is the same as

$$
A_{1}(\tilde{u}, \phi)=\left(k^{2}-1\right) B(\tilde{u}, \phi)
$$

Thus, $\tilde{u}=\left(k^{2}-1\right) T \tilde{u}$.

Suppose, conversely, that $\tilde{u} \in H^{1}\left(\mathbb{R}^{3}\right)$ is an eigenfunction for $T$ with eigenvalue $\lambda$. Then $\tilde{u}$ satisfies (2.17). By Theorem 2.2, $\tilde{u}$ can be written as a series (2.10) for $|x| \geq r_{0}$. Proposition 2.5 implies that $\tilde{u}$ is outgoing. Then $u=u(\tilde{u})$ satisfies (2.1) with $f=0$ and is also outgoing. This completes the proof of the theorem.

Remark 2.7. It is clear that the PML method only gives the resonances which satisfy $\operatorname{Im}\left(d_{0} k\right)>0$, i.e., those which are in the sector bounded by the positive real axis and the line $\arg (z)=\arg \left(1 / d_{0}\right)$. To get the resonances to the left of this line, we need to increase $\sigma_{0}$.

The Aguilar-Balslev-Combes-Simon theory is part of spectral deformation theory [16] which provides additional information about the spectrum of the PML operator $\widetilde{L}$. Specifically, these results imply that the essential spectrum of $\widetilde{L}$ is

$$
\sigma_{\text {ess }}(\widetilde{L})=\left\{z \mid \arg (z)=-2 \arg \left(1+i \sigma_{0}\right)\right\}
$$

(cf. Theorem 18.6 of [16]). This implies that the eigenvalues of $\widetilde{L}$ corresponding to resonances are isolated and of finite multiplicity. Note that if $z$ is in $\sigma_{\text {ess }}(\widetilde{L})$, $\operatorname{Im}\left(d_{0} k\right)=0$.

\section{The truncated PML PRoblem}

We shall show that the PML eigenfunctions and generalized eigenfunctions decay exponentially later. It is then natural to approximate them on a bounded computational domain with a convenient boundary condition. To this end, we introduce a bounded (computational) domain $\Omega_{\delta}$ whose boundary is denoted by $\Gamma_{\delta}$. We shall always assume that the transition layer is in $\Omega_{\delta}$, i.e., $\Omega_{1} \subset \Omega_{\delta}$. We assume that the outer boundary of $\Omega_{\delta}$ is given by dilation of a fixed boundary by a parameter $\delta$, e.g., $\Omega_{\delta}$ is a cube of side length $2 \delta$ (see Figure 1). We also assume that $\Omega_{\delta}$ is convex.

The techniques of [9] can be used to show that the sesquilinear form $A_{1}(\cdot, \cdot)$ still satisfies an inf-sup condition on $H_{0}^{1}\left(\Omega_{\delta}\right)$ provided that $\delta \geq \delta_{0}$ and $\delta_{0}$ is sufficiently large; i.e., for $u \in H_{0}^{1}\left(\Omega_{\delta}\right)$,

$$
\|u\|_{H^{1}\left(\Omega_{\delta}\right)} \leq C \sup _{\phi \in H_{0}^{1}\left(\Omega_{\delta}\right)} \frac{\left|A_{1}(u, \phi)\right|}{\|\phi\|_{H^{1}\left(\Omega_{\delta}\right)}} .
$$

The analogous inf-sup condition for the adjoint operator holds as well:

$$
\|\phi\|_{H^{1}\left(\Omega_{\delta}\right)} \leq C \sup _{u \in H_{0}^{1}\left(\Omega_{\delta}\right)} \frac{\left|A_{1}(u, \phi)\right|}{\|u\|_{H^{1}\left(\Omega_{\delta}\right)}} .
$$

Here and in the remainder of this paper, $C$ is independent of $\delta$ once $\delta$ is sufficiently large. 
Because of the above inf-sup condition, we can define the operator $T_{\delta}: H^{1}\left(\mathbb{R}^{3}\right) \rightarrow$ $H_{0}^{1}\left(\Omega_{\delta}\right) \subset H^{1}\left(\mathbb{R}^{3}\right)$ by $T_{\delta} f=u$, where $u \in H_{0}^{1}\left(\Omega_{\delta}\right)$ is the unique solution to

$$
A_{1}(u, \phi)=B(f, \phi) \text { for all } \phi \in H_{0}^{1}\left(\Omega_{\delta}\right) .
$$

Our goal will be to study how the spectrum of $T_{\delta}$ is related to that of $T$. Our first theorem shows that the resolvent set for $T_{\delta}$ approaches that of $T$ as $\delta$ becomes large. This means that the truncated problem does not result in spurious eigenvalues in the region of interest, $\operatorname{Im}\left(d_{0} k\right)>0$.

Theorem 3.1. Let $U$ be a compact subset of $\rho(T)$, the resolvent set of $T$, whose image under the map $z \mapsto \sqrt{(1+z) / z} \equiv k(z)$ satisfies $\operatorname{Im}\left(d_{0} k(z)\right)>0$ for all $z \in U$. Here we have taken $-\pi<\arg (k(z)) \leq 0$. Then, there exists a $\delta_{0}$ (depending on $U$ ) such that for $\delta>\delta_{0}, U \subset \rho\left(T_{\delta}\right)$.

We shall need the following proposition for the proof of the above theorem. Its proof is given in the Appendix.

Proposition 3.2. Assume that $w$ is in $H^{1}\left(\mathbb{R}^{3}\right)$ and satisfies (2.15) in $\Omega_{1}^{c}$ with $\beta^{2}=d_{0}^{2} k(z)^{2}$ and $z \in U$ as in Theorem 3.1. Then there is a positive number $\alpha$ and $\delta_{0}>r_{1}$ such that for $\delta \geq \delta_{0}$,

$$
\|w\|_{H^{1 / 2}\left(\Gamma_{\delta}\right)} \leq C e^{-\alpha \delta}\|w\|_{H^{1 / 2}\left(\Gamma_{1}\right)} .
$$

The constants $C$ and $\alpha$ can be taken independent of $z \in U$ and $\delta \geq \delta_{0}$.

Proof of Theorem 3.1. Let $R_{z}(T)=(T-z I)^{-1}$ be the resolvent operator and $\left\|R_{z}(T)\right\|_{H^{1}\left(\mathbb{R}^{3}\right)}$ denote its operator norm. This norm depends continuously for $z \in \rho(T)$, so there is a constant $C=C_{U}$ such that

$$
\left\|R_{z}(T)\right\|_{H^{1}\left(\mathbb{R}^{3}\right)} \leq C \text { for all } z \in U .
$$

For $u \in H^{1}\left(\mathbb{R}^{3}\right)$, set $\phi=(T-z I) u$. Then for $z \in U$, using (2.13),

$$
\begin{aligned}
\|u\|_{H^{1}\left(\mathbb{R}^{3}\right)} & \leq C\|\phi\|_{H^{1}\left(\mathbb{R}^{3}\right)} \leq C \sup _{v \in H^{1}\left(\mathbb{R}^{3}\right)} \frac{\left|A_{1}(\phi, v)\right|}{\|v\|_{H^{1}\left(\mathbb{R}^{3}\right)}} \\
& =C \sup _{v \in H^{1}\left(\mathbb{R}^{3}\right)} \frac{\left|\widetilde{A}_{z}(u, v)\right|}{\|v\|_{H^{1}\left(\mathbb{R}^{3}\right)}} .
\end{aligned}
$$

Here we have set $\widetilde{A}_{z}(\cdot, \cdot) \equiv B(\cdot, \cdot)-z A_{1}(\cdot, \cdot)$. The inf-sup condition for the adjoint holds as well by similar reasoning.

We will show that the corresponding inf-sup conditions on the truncated domain hold for all $z \in U$ if $\delta_{0}$ is large enough. Namely, for $u \in H_{0}^{1}\left(\Omega_{\delta}\right)$,

$$
\|u\|_{H^{1}\left(\Omega_{\delta}\right)} \leq C \sup _{v \in H_{0}^{1}\left(\Omega_{\delta}\right)} \frac{\left|\widetilde{A}_{z}(u, v)\right|}{\|v\|_{H^{1}\left(\Omega_{\delta}\right)}}
$$

and

$$
\|u\|_{H^{1}\left(\Omega_{\delta}\right)} \leq C \sup _{v \in H_{0}^{1}\left(\Omega_{\delta}\right)} \frac{\left|\widetilde{A}_{z}(v, u)\right|}{\|v\|_{H^{1}\left(\Omega_{\delta}\right)}} .
$$

Once we show (3.4) and (3.5), it then follows that the solution $v \in H_{0}^{1}\left(\Omega_{\delta}\right)$ to the variational problem

$$
\widetilde{A}_{z}(v, \phi)=A_{1}(w, \phi) \text { for all } \phi \in H_{0}^{1}\left(\Omega_{\delta}\right)
$$


satisfies

$$
\left(T_{\delta}-z I\right) v=w
$$

This shows that $z$ is in $\rho\left(T_{\delta}\right)$.

We start with (3.3) to verify (3.4). The test function $v$ appearing in (3.3) is decomposed as $v=v_{0}+v_{1}$, where $v_{1}$ is defined on all of $\mathbb{R}^{3}$ by

$$
\begin{aligned}
\widetilde{A}_{z}\left(\chi, v_{1}\right) & =0 \text { for all } \chi \in H_{0}^{1}\left(\Omega_{\delta} \backslash \bar{\Omega}_{1}\right), \\
v_{1} & =0 \text { on } \Omega_{1}, \\
v_{1} & =v \text { on } \Omega_{\delta}^{c} .
\end{aligned}
$$

The problem defining $v_{1}$ on $\Omega_{\delta} \backslash \bar{\Omega}_{1}$ is uniquely solvable. Indeed, let $\chi \in H_{0}^{1}\left(\Omega_{\delta} \backslash \bar{\Omega}_{1}\right)$ and $\gamma$ be in $\mathbb{C}$. Then

$$
\widetilde{A}_{z}(\gamma \chi, \chi)=z\left(\gamma d_{0}^{2} k(z)^{2}(\chi, \chi)-\gamma D(\chi, \chi)\right) .
$$

Here $D(\cdot, \cdot)$ denotes the Dirichlet form. Now, since $U$ is compact, there is an $\epsilon$ with $0<\epsilon<\pi$ such that $\epsilon<\arg \left(d_{0}^{2} k(z)^{2}\right)<\pi$ for all $z \in U$. Taking $\gamma=\exp (-i \epsilon / 2)$ above implies that both $-\gamma$ and $\gamma d_{0}^{2} k(z)^{2}$ have a positive imaginary part. It follows that

$$
\left|\widetilde{A}_{z}(\gamma \chi, \chi)\right| \geq C\|\chi\|_{H^{1}\left(\Omega_{\delta} \backslash \bar{\Omega}_{1}\right)}^{2}
$$

The unique solvability of (3.7) follows, and we have

$$
\left\|v_{1}\right\|_{H^{1}\left(\mathbb{R}^{3}\right)} \leq C\|v\|_{H^{1}\left(\mathbb{R}^{3}\right)} .
$$

Next for $u \in H_{0}^{1}\left(\Omega_{\delta}\right)$ we write $u=u_{0}+u_{1}$, where

$$
\begin{aligned}
\widetilde{A}_{z}\left(u_{1}, \chi\right) & =0 \text { for all } \chi \in H_{0}^{1}\left(\Omega_{\delta} \backslash \bar{\Omega}_{1}\right), \\
u_{1} & =u \text { on } \Omega_{1}, \\
u_{1} & =0 \text { on } \Omega_{\delta}^{c} .
\end{aligned}
$$

As above, the problem defining $u_{1}$ on $\Omega_{\delta} \backslash \bar{\Omega}_{1}$ is also uniquely solvable and

$$
\left\|u_{1}\right\|_{H^{1}\left(\mathbb{R}^{3}\right)} \leq C\|u\|_{H^{1}\left(\Omega_{\delta}\right)} .
$$

We then have

$$
\begin{aligned}
\widetilde{A}_{z}(u, v) & =\widetilde{A}_{z}\left(u, v_{0}\right)+\widetilde{A}_{z}\left(u_{0}, v_{1}\right)+\widetilde{A}_{z}\left(u_{1}, v_{1}\right) \\
& =\widetilde{A}_{z}\left(u, v_{0}\right)+\widetilde{A}_{z}\left(u_{1}, v_{1}\right) .
\end{aligned}
$$

Now, let $\tilde{u}_{1}$ in $H^{1}\left(\mathbb{R}^{3}\right)$ solve

$$
\begin{aligned}
\tilde{A}_{z}\left(\tilde{u}_{1}, \eta\right) & =0 \text { for all } \eta \in H_{0}^{1}\left(\Omega_{1}^{c}\right) \\
\tilde{u}_{1} & =u \text { on } \Omega_{1} .
\end{aligned}
$$

The argument showing unique solvability of (3.7) works as well here.

We then have

$$
\widetilde{A}_{z}\left(u_{1}, v_{1}\right)=\widetilde{A}_{z}\left(u_{1}-\tilde{u}_{1}, v_{1}\right)+\widetilde{A}_{z}\left(\tilde{u}_{1}, v_{1}\right)=\widetilde{A}_{z}\left(u_{1}-\tilde{u}_{1}, v_{1}\right) .
$$

Now $u_{1}-\tilde{u}_{1}$ satisfies $u_{1}-\tilde{u}_{1}=0$ on $\Omega_{1}$ and

$$
\widetilde{A}_{z}\left(u_{1}-\tilde{u}_{1}, \phi\right)=0 \text { for all } \phi \in H_{0}^{1}\left(\Omega_{\delta} \backslash \bar{\Omega}_{1}\right) \oplus H_{0}^{1}\left(\Omega_{\delta}^{c}\right),
$$

with $u_{1}-\tilde{u}_{1}=0$ on $\Gamma_{1}$ and $u_{1}-\tilde{u}_{1}=-\tilde{u}_{1}$ on $\Gamma_{\delta}$. By the stability of the problem (3.9) on $H_{0}^{1}\left(\Omega_{\delta} \backslash \bar{\Omega}_{1}\right)$ and $H_{0}^{1}\left(\Omega_{\delta}^{c}\right)$, and by Proposition 3.2.

$$
\left\|u_{1}-\tilde{u}_{1}\right\|_{H^{1}\left(\mathbb{R}^{3}\right)} \leq C\left\|\tilde{u}_{1}\right\|_{H^{1 / 2}\left(\Gamma_{\delta}\right)} \leq C e^{-\alpha \delta}\|u\|_{H^{1 / 2}\left(\Gamma_{1}\right)} .
$$


It then follows from (3.8) and a standard trace estimate that

$$
\left|\widetilde{A}_{z}\left(u_{1}, v_{1}\right)\right| \leq C e^{-\alpha \delta}\|u\|_{H^{1}\left(\Omega_{\delta}\right)}\|v\|_{H^{1}\left(\mathbb{R}^{3}\right)} .
$$

Thus,

$$
\|u\|_{H^{1}\left(\Omega_{\delta}\right)} \leq C \sup _{v_{0} \in H_{0}^{1}\left(\Omega_{\delta}\right)} \frac{\left|\widetilde{A}_{z}\left(u, v_{0}\right)\right|}{\left\|v_{0}\right\|_{H^{1}\left(\Omega_{\delta}\right)}}+C e^{-\alpha \delta}\|u\|_{H^{1}\left(\Omega_{\delta}\right)} .
$$

The inf-sup condition (3.4) follows by taking $\delta_{0}$ large enough so that $C e^{-\alpha \delta_{0}}<1$. The proof of (3.5) is similar. This completes the proof of the theorem.

\section{Eigenvalue COnVERGence}

We study eigenvalue convergence in this section. As mentioned above, the eigenvalues of $T$ corresponding to resonances are isolated and of finite multiplicity. Let $\lambda$ be such an eigenvalue. Since $\lambda$ is isolated, there is a neighborhood of it with all points excluding $\lambda$ in $\rho(T)$. Let $\eta>0$ be such that the circle of radius $\eta$ centered at $\lambda$ is in this neighborhood. We denote this circle by $\Gamma$.

The following projector will play a major role in the eigenvalue convergence analysis: For $u \in H^{1}\left(\mathbb{R}^{3}\right)$, define

$$
P_{\Gamma}(u)=\frac{1}{2 \pi i} \int_{\Gamma} R_{z}(T) u d z .
$$

Its range is denoted by $V$ and is the generalized eigenspace associated with the eigenvalue $\lambda$.

By Theorem 3.1, $\rho\left(T_{\delta}\right)$ contains $\Gamma$ for sufficiently large $\delta$. For such $\delta$, we define the corresponding projector:

$$
P_{\Gamma}^{\delta}(u)=\frac{1}{2 \pi i} \int_{\Gamma} R_{z}\left(T_{\delta}\right) u d z .
$$

Its range will be denoted by $V_{\delta}$, which is the subspace of $H_{0}^{1}\left(\Omega_{\delta}\right)$ spanned by the generalized eigenfunctions associated with the eigenvalues of $T_{\delta}$ inside $\Gamma$. A main result of this paper is contained in the following theorem.

Theorem 4.1. For any $\eta$ sufficiently small, there is a $\delta_{1}>0$ such that

$$
\operatorname{dim}(V)=\operatorname{dim}\left(V_{\delta}\right)
$$

for $\delta>\delta_{1}$.

Remark 4.2. The above theorem shows that the eigenvalues for the truncated problem converge to those of the full problem. This convergence respects the eigenvalue multiplicity in the sense that the sum of the multiplicities of the eigenvalues inside the circle of radius $\eta$ for the truncated problem equals the multiplicity of $\lambda$ for any $\eta$ provided that $\delta \geq \delta_{1}(\eta)$ is sufficiently large.

The typical approach for proving eigenvalue convergence results such as Theorem 4.1 involves showing that the approximate operator $\left(T_{\delta}\right.$ in this case) converges in norm to the full operator. This fails for our application as $T_{\delta}$ cannot converge to $T$ in norm since $T_{\delta}$ is compact for any $\delta$ while $T$ is not. Moreover, $T_{\delta}$ is not even norm continuous as a function of $\delta$.

Instead, our eigenvalue convergence analysis is based on the exponential decay of the generalized eigenfunctions in both $V$ and $V_{\delta}$. The main technical results are contained in the following three lemmas whose proofs are given in the Appendix. 
Lemma 4.3. Let $V$ be as above. Then there are constants $\alpha, C$ and $M>r_{1}$ such that for all $\psi \in V$,

$$
|\psi(x)| \leq C e^{-\alpha|x|}\|\psi\|_{H^{1}\left(\mathbb{R}^{3}\right)} \text { for }|x|>M .
$$

As $T_{\delta}$ is compact, the generalized eigenspace $V_{\delta}$ has a finite dimension and a basis of the form $\psi_{i, j}, i=1, \ldots, k, j=1, \ldots, m(i)$. Here if $\lambda_{i}^{\delta}$ is an eigenvalue of $T_{\delta}$ inside $\Gamma$ for $i=1, \ldots, k$ we may take

$$
\psi_{i, j}=\left(T_{\delta}-\lambda_{i}^{\delta}\right) \psi_{i, j+1} \quad \text { and } \quad\left(T_{\delta}-\lambda_{i}^{\delta}\right) \psi_{i, 1}=0 .
$$

A priori we do not have a bound on the dimension of $V_{\delta}$. To deal with this, we consider subspaces $\widetilde{V}_{\delta}$ of $V_{\delta}$ of dimension at most $\operatorname{dim}(V)+1$. Specifically, let $\widetilde{V}_{\delta}$ have a basis of the form $\left\{\psi_{i, j}\right\}, \psi_{i, j}, i=1, \ldots, k, j=1, \ldots, \tilde{m}(i)$ with $\left\{\psi_{i, j}\right\}$ as above and $\sum_{i} \tilde{m}(i) \leq \operatorname{dim}(V)+1$. The space $\widetilde{V}_{\delta}$ is invariant under $T_{\delta}$ and $P_{\Gamma}^{\delta}$. The following lemma gives a decay estimate for functions in $\widetilde{V}_{\delta}$. The constant can be taken so that it only depends on the dimension of $V$, provided that $\delta$ is large enough.

Lemma 4.4. Let $\widetilde{V}_{\delta}$ be as above. Then there are constants $\alpha, C$ and $M>r_{1}$ such that for $\delta>M$ and $\psi_{\delta} \in \widetilde{V}_{\delta}$,

$$
\left|\psi_{\delta}(x)\right| \leq C e^{-\alpha|x|}\left\|\psi_{\delta}\right\|_{H^{1}\left(\Omega_{\delta}\right)} \text { for }|x|>M .
$$

Lemma 4.5. Suppose that $u \in H^{1}\left(\mathbb{R}^{3}\right)$ satisfies

$$
|u(x)| \leq C e^{-\alpha|x|}\|u\|_{H^{1}\left(\mathbb{R}^{3}\right)}
$$

for $|x|>M>r_{1}$. Then there exist positive constants $\alpha_{1}, C_{1}$ and $M_{1}>M$ such that

$$
\left\|\left(T-T_{\delta}\right) u\right\|_{H^{1}\left(\mathbb{R}^{3}\right)} \leq C_{1} e^{-\alpha_{1} \delta}\|u\|_{H^{1}\left(\mathbb{R}^{3}\right)}
$$

for $\delta>M_{1}$.

Proof of Theorem 4.1. We first note that for $z \in \Gamma$,

$$
\left\|R_{z}(T)\right\| \leq C .
$$

In addition, for $\delta>\delta_{0}$ in Theorem 3.1, (3.4) and (3.5) implies that

$$
\left\|R_{z}\left(T_{\delta}\right)\right\| \leq C,
$$

with $C$ independent of $\delta$. It follows that $P_{\Gamma}$ and $P_{\Gamma}^{\delta}$ are bounded operators in $H^{1}\left(\mathbb{R}^{3}\right)$.

Let $\psi$ be in $V$. Since $V$ is invariant under the action of $R_{z}(T)$, by Lemma 4.5 ,

$$
\left\|\left(T-T_{\delta}\right) R_{z}(T) \psi\right\|_{H^{1}\left(\mathbb{R}^{3}\right)} \leq C e^{-\alpha_{1} \delta}\|\psi\|_{H^{1}\left(\mathbb{R}^{3}\right)} .
$$

We also have

$$
\begin{aligned}
\left(I-P_{\Gamma}^{\delta}\right) P_{\Gamma} & =\frac{1}{2 \pi i} \int_{\Gamma}\left(R_{z}(T)-R_{z}\left(T_{\delta}\right)\right) P_{\Gamma} d z \\
& =-\frac{1}{2 \pi i} \int_{\Gamma} R_{z}\left(T_{\delta}\right)\left(T-T_{\delta}\right) R_{z}(T) P_{\Gamma} d z
\end{aligned}
$$




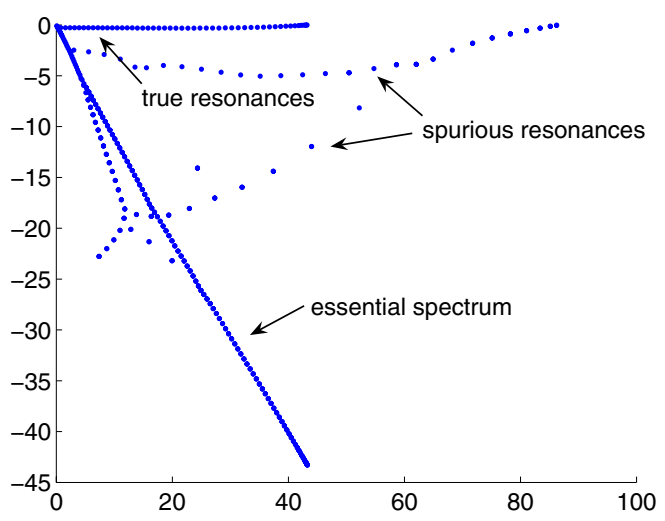

Figure 2. Spectrum of a one-dimensional resonance problem

Since $P_{\Gamma} \psi=\psi$, we have

$$
\begin{aligned}
\left\|\left(I-P_{\Gamma}^{\delta}\right) \psi\right\|_{H^{1}\left(\mathbb{R}^{3}\right)} & =\frac{1}{2 \pi}\left\|\int_{\Gamma} R_{z}\left(T_{\delta}\right)\left(T-T_{\delta}\right) R_{z}(T) \psi d z\right\|_{H^{1}\left(\mathbb{R}^{3}\right)} \\
& \leq \frac{1}{2 \pi} \int_{\Gamma}\left\|R_{z}\left(T_{\delta}\right)\right\|_{H^{1}\left(\mathbb{R}^{3}\right)}\left\|\left(T-T_{\delta}\right) R_{z}(T) \psi\right\|_{H^{1}\left(\mathbb{R}^{3}\right)} d z \\
& \leq C e^{-\alpha_{1} \delta}\|\psi\|_{H^{1}\left(\mathbb{R}^{3}\right)} .
\end{aligned}
$$

We choose $\delta_{1} \geq \delta_{0}$ so that $C e^{-\alpha \delta_{1}}$ is less than one. For (4.6) to hold, it is necessary that the rank of $P_{\Gamma}^{\delta}$ be greater than or equal to $\operatorname{dim}(V)$, i.e., $\operatorname{dim}\left(V_{\delta}\right) \geq \operatorname{dim}(V)$.

For the opposite inequality, we let $\psi$ be in $\widetilde{V}_{\delta}$ with $\widetilde{V}_{\delta}$ as above. An argument similar to that used above (using the invariance of $\widetilde{V}_{\delta}$ under $P_{\Gamma}^{\delta}$ ) gives

$$
\left\|\left(I-P_{\Gamma}\right) \psi\right\|_{H^{1}\left(\mathbb{R}^{3}\right)} \leq C e^{-\alpha \delta}\|\psi\|_{H^{1}\left(\mathbb{R}^{3}\right)} .
$$

Choosing $\delta_{1} \geq \delta_{0}$ so that $C e^{-\alpha \delta_{1}}<1$ then leads to $\operatorname{dim}(V) \geq \operatorname{dim}\left(\widetilde{V}_{\delta}\right)$. This implies that there is no subspace $\widetilde{V}_{\delta} \subseteq V_{\delta}$ with dimension greater than $\operatorname{dim}(V)$, i.e., $\operatorname{dim}\left(V_{\delta}\right)=\operatorname{dim}(V)$.

\section{Numerical EXPERIMENTS}

In this section, we will give simple one and two-dimensional resonance problems illustrating the behavior of finite element approximations of the PML eigenvalue problem. Although some experiments appear to have spurious numerical eigenvalues, we shall see that they can be controlled by keeping the transition layer close to the non-homogeneous phenomena, i.e. the region where the operator differs from the Laplacian.

We start with a one dimensional problem, i.e.,

$$
-a \Delta u=k^{2} u \text { in } \mathbb{R}
$$

with the outgoing wave condition. Here $a$ is a piecewise constant function defined by

$$
a=\left\{\begin{array}{cl}
1 / 4 & \text { if }|x|<1 \\
1 & \text { otherwise }
\end{array}\right.
$$


We impose the continuity of $u$ and $a u^{\prime}$ at $x= \pm 1$. The analytic resonances corresponding to this problem are given by

$$
k=\frac{n \pi}{4}-\frac{\ln 3}{4} i
$$

for $n \in \mathbb{Z}$ and $n>0$.

For the first experiment, we choose the PML parameters $r_{0}=2, r_{1}=4$, $\delta=8, \sigma_{0}=1$ and discretize the system with a mesh size $h=1 / 50$. Figure 2 shows the resulting eigenvalues. Note that the eigenvalues labeled "true resonances" are very close to the analytic resonances given above. In Figure 3, we report the error observed when approximating the resonance of smallest magnitude as a function of $\delta$ for fixed values of $h$. The PML parameters were $r_{0}=1, r_{1}=2$, and $\sigma_{0}=1$. As expected, increasing $\delta$ for a fixed value of $h$ improves the accuracy to the point where the mesh size errors dominate.

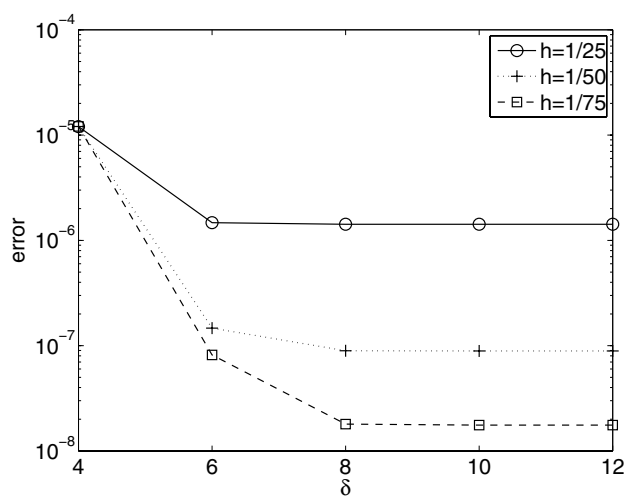

FiguRE 3. Eigenvalue error for the resonance of smallest magnitude

The remaining eigenvalues in Figure 2 either correspond to those clearly approximating the essential spectrum or to spurious eigenvalues. Those far away from the true resonances and those to the left of the essential spectrum are easily ignored. However, the group of spurious eigenvalues running below and parallel to the true resonances are somewhat disturbing, especially since they do not move much when either the mesh size is decreased (at least, within reasonable parameters) or the computational region is increased.

Spurious eigenvalues appearing in PML approximations to resonance problems have been discussed elsewhere in the literature. In particular, Zworski 26] explains this phenomenon in terms of the pseudo-spectra concept (cf. [25]). Unlike symmetric matrices, the norms of the resolvent of a non-symmetric operator can be quite large for points located far away from the spectrum. We shall demonstrate that this is an issue for the PML eigenvalue problem. Note that the central theorems (Theorem 3.1 and Theorem 4.1) of this paper require that $\delta$ is large enough that

$$
C\left(\left\|R_{z}(T)\right\|\right) e^{-\alpha_{1} \delta}<1 .
$$

The situation at the discrete level is worse. In fact, to guarantee eigenvalue convergence without spurious eigenvalues from the discretization, one needs to have that 


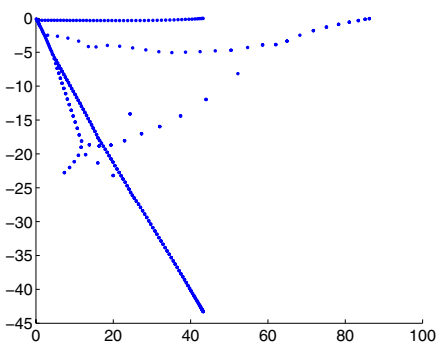

(a) $r_{0}=2$

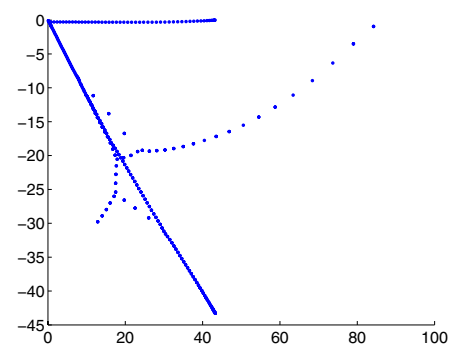

(c) $r_{0}=1.2$

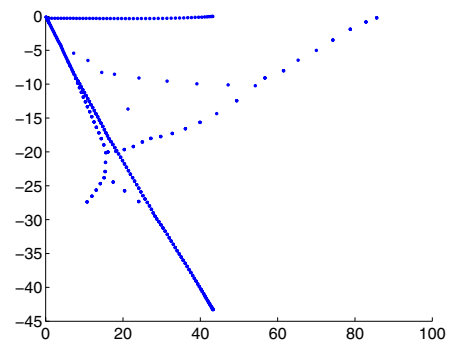

(b) $r_{0}=1.5$

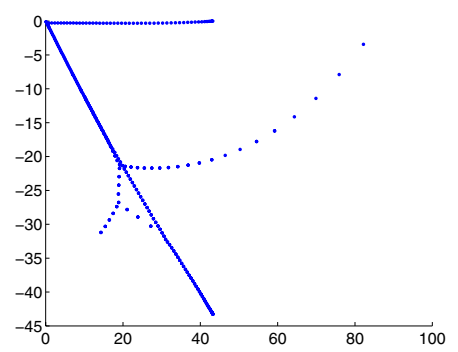

(d) $r_{0}=1$

Figure 4. Eigenvalues from different PML's. $r_{0}$ is the radius of the inside boundary of the PML

$h \leq h_{0}$ with $h_{0}$ satisfying

$$
C\left(\left\|R_{z}\left(T_{\delta}\right)\right\|\right) h_{0}<1
$$

Thus, in cases where the norm of the resolvent is large, to get rid of the spurious eigenvalues, it appears to be necessary to make $h$ too small to be practical.

To shed some light on the behavior of the resolvent, we consider the above onedimensional problem. Let $k$ be a complex number with $\operatorname{Im}(k)<0, \operatorname{Im}\left(d_{0} k\right)>0$ and $k$ not a resonance. The function $f(x)=e^{i k \tilde{d}|x|}$ satisfies the PML equation

$$
\left(\widetilde{L}-k^{2}\right) f \equiv-\frac{1}{d}\left(\frac{1}{d} f^{\prime}\right)^{\prime}-k^{2} f=0 \text { for }|x|>1 .
$$

Note that with $r_{0}>1, f$ increases exponentially from $|x|=1$ to $|x|=r_{0}$ while decreasing exponentially outside of the transition region. Because $f$ is relatively small for $|x| \leq 1$ and satisfies (5.3) $),\left\|\left(\widetilde{L}-k^{2}\right) f\right\|$ is much smaller than $\|f\|$. This implies that the constant in the inf-sup condition (3.3) is large. This constant is directly proportional to the norm of the resolvent $R_{z}(T)$ (see the proof of Theorem 3.1 for details). Accordingly, to keep the norm of $R_{z}(T)$ manageable, we need to avoid a large region allowing an exponential increase. This can be attained by keeping the start of the transitional region as close as possible to the region of inhomogeneity, i.e., $|x|=1$. The analysis for problems of dimension greater than one is similar.

The behavior of the spurious resonances as a function of the location of the transitional layer is illustrated in Figure 4. Notice that the spurious eigenvalues 
can be moved away from the true resonances simply by placing the transition region closer to one. In fact, the best results are obtained by starting the transition region on the interface. This also illustrates the fact that there does not need to be any area extending outside of $\Omega$ where the original equation is retained.

We next consider a model problem on $\mathbb{R}^{2}$. Let $\Omega_{0}$ be the open unit disc in $\mathbb{R}^{2}$ and consider

$$
-a \Delta u=k^{2} u \text { in } \mathbb{R}^{2}
$$

with the outgoing wave condition and the transmission conditions of the continuity of $u$ and $a \nabla u$ at the interface, where

$$
a=\left\{\begin{array}{cl}
1 / 4 & \text { if }(x, y) \in \Omega_{0} \\
1 & \text { otherwise }
\end{array}\right.
$$

An outgoing solution bounded in $\Omega_{0}$ is of the form (in polar coordinates)

$$
u(x, y)=\left\{\begin{array}{l}
\sum_{n=-\infty}^{\infty} a_{n} J_{n}(2 k r) e^{i n \theta} \text { for }(x, y) \in \Omega_{0}, \\
\sum_{n=-\infty}^{\infty} b_{n} H_{n}^{1}(k r) e^{i n \theta} \text { for }(x, y) \in \Omega_{0}^{c},
\end{array}\right.
$$

where $J_{n}$ are Bessel functions of the first kind of order $n$ and $H_{n}^{1}$ are Hankel functions of the first kind of order $n$. The continuity conditions at the interface lead to

$$
a_{n} J_{n}(2 k)=b_{n} H_{n}^{1}(k) \text { and } \frac{1}{2} a_{n} J_{n}^{\prime}(2 k)=b_{n}\left(H_{n}^{1}(k)\right)^{\prime} .
$$

Non-zero solutions exist when $k$ satisfies

$$
J_{n}^{\prime}(2 k) H_{n}^{1}(k)-2 J_{n}(2 k)\left(H_{n}^{1}(k)\right)^{\prime}=0 .
$$

This equation can be solved by iteration, and its solutions are used as a reference. It is easy to see that each solution $k$ to the problem (5.4) for $n>0$ is of multiplicity 2 .

For the one-dimensional case, we simply computed all eigenvalues using MatLab. This approach fails for the two-dimensional problem, as the problem size is much too large. We clearly have to be more selective. Our goal is to focus on computing the eigenvalues corresponding to resonances which are close to the origin. We are able to do this by defining a related eigenvalue problem which transforms the eigenvalues of interest into the eigenvalues of greatest magnitude. These eigenvalues can then be selectively computed using a general eigensolver software. Specifically, we use the software package SLEPc [15], which is a general purpose eigensolver built on top of PETSc [2.

The computational eigenvalue problem (after introducing PML, truncating the domain and applying finite elements) can be written as

$$
S u=k^{2} N u
$$

for appropriate complex valued matrices $S$ and $N$. The idea is to use linear fractional transformations. We consider $\zeta_{2} \circ \zeta_{1}$, where

$$
\zeta_{1}(z)=\frac{1}{z} \quad \text { and } \quad \zeta_{2}(z)=\frac{d_{0}+i z}{d_{0}-i z}
$$

The first transformation maps points near the origin to points of large absolute value. Under this transformation, the sector $2 \arg \left(1 / d_{0}\right) \leq \arg (z) \leq 0 \operatorname{maps}$ 
to the sector $0 \leq \arg (z) \leq 2 \arg \left(d_{0}\right)$. The second transformation gets rid of the "essential spectrum" (which was mapped to $\arg (z)=2 \arg \left(d_{0}\right)$ ) by mapping $\arg (z)=2 \arg \left(d_{0}\right)$ to the interior of the unit disk and anything in the sector $0 \leq \arg (z)<\arg \left(d_{0}\right)$ to the exterior of the unit disk. Thus, we look for the eigenvalues of largest magnitude for the operator

$$
\left(d_{0} S+i N\right) u=\mu\left(d_{0} S-i N\right) u
$$

and recover $k^{2}$ from the formula

$$
k^{2}=\frac{(\mu+1) i}{(\mu-1) d_{0}} .
$$

We consider computing on a square domain of side length $2 \delta$. Table 1 gives the values of the first ten numerical and analytical resonances for the above problem as a function of $h$. The truncated domain corresponded to $\delta=5$ and the PML parameters were $\sigma_{0}=1, r_{0}=1$ and $r_{1}=4$. Note that errors of less than one percent were obtained and that improved results were observed when the mesh size was decreased. These are relatively large problems; indeed, the case of $h=1 / 120$

\begin{tabular}{|c|c|c|c|c|}
\hline \multicolumn{2}{|c|}{ Approximate PML Resonances } & \multirow{2}{*}{ Resonances } & \multirow{2}{*}{ Multiplicity } & \multirow{2}{*}{$n$} \\
\hline$h=1 / 100$ & $h=1 / 120$ & & & \\
\hline $1.1169-0.2393 i$ & $1.1165-0.2392 i$ & $1.1155-0.2396 i$ & 1 & 0 \\
\hline $2.7211-0.2667 i$ & $2.7200-0.2665 i$ & $2.7167-0.2665 i$ & 1 & 0 \\
\hline $1.8264-0.2916 i$ & $1.8256-0.2914 i$ & & 2 & \\
\hline $1.8264-0.2916 i$ & $1.8256-0.2914 i$ & $1.8200-0.2921 \mathrm{l}$ & 2 & 1 \\
\hline $2.4021-0.3759 i$ & $2.4009-0.3755 i$ & $2.3981-0.3781 i$ & 2 & 2 \\
\hline $2.4026-0.3761 i$ & $2.4012-0.3757 i$ & & & \\
\hline $2.8249-0.3182 i$ & $2.8242-0.3173 i$ & $28161-03161 i$ & 2 & 3 \\
\hline $2.8249-0.3182 i$ & $2.8242-0.3173 i$ & & & \\
\hline $3.4066-0.1881 i$ & $3.4058-0.1877 i$ & $3.3993-0.18$ & 2 & 4 \\
\hline $3.4068-0.1885 i$ & $3.4059-0.1880 i$ & & & 4 \\
\hline
\end{tabular}
corresponds to almost a million and a half complex unknowns.

TABLE 1. Numerical results for the first ten resonances of the twodimensional problem

\section{Appendix}

The main results of this Appendix are the exponential decay of eigenfunctions for the full and truncated PML problems. We also prove other lemmas and propositions which have appeared earlier without proof. We start with the following lemma.

Lemma 6.1. Suppose that $w$ is in $H^{1}\left(\Omega_{1}^{c}\right)$ and satisfies

$$
\Delta w+\beta^{2} w=f \text { in } \Omega_{1}^{c}
$$

with $\operatorname{Im}(\beta)$ positive and $f \in L^{2}\left(\Omega_{1}^{c}\right)$. If $f$ decays exponentially, i.e., there are positive constants $\alpha, C_{f}$ and $M>r_{1}$ such that $|f(x)| \leq C_{f} e^{-\alpha|x|}$ for $|x|>M$, then there are positive constants $\alpha_{1}, C_{1}$ and $M_{1}>M$ such that

$$
|w(x)| \leq C_{1} e^{-\alpha_{1}|x|}\left(\|w\|_{H^{1}\left(\Omega_{1}^{c}\right)}+\|f\|_{L^{2}\left(\Omega_{1}^{c}\right)}+C_{f}\right)
$$


and

$$
\|w\|_{H^{1 / 2}\left(\Gamma_{\delta}\right)} \leq C_{1} e^{-\alpha_{1} \delta}\left(\|w\|_{H^{1}\left(\Omega_{1}^{c}\right)}+\|f\|_{L^{2}\left(\Omega_{1}^{c}\right)}+C_{f}\right)
$$

for $|x|, \delta>M_{1}$. Here $\alpha_{1}, C_{1}$ and $M_{1}$ can be chosen independent of $w, f$ and $\delta$.

Proof. Choose any $\tilde{M}_{1}>M$. For $|x|>\tilde{M}_{1}$ let $\Omega_{M}$ and $\Omega_{R}$ be open balls centered at the origin of radius $M$ and $2|x|$, respectively. Let $\Gamma_{M}$ and $\Gamma_{R}$ denote their boundaries.

By Green's theorem, we have for $|x|>\tilde{M}_{1}$

$$
w(x)=-\int_{\Gamma_{M} \cup \Gamma_{R}}\left[\frac{\partial w}{\partial n}(y) \Phi(x, y)-w(y) \frac{\partial \Phi}{\partial n_{y}}(x, y)\right] d S_{y}+\int_{D} f(y) \Phi(x, y) d y
$$

where $n$ is the outward normal vector on the boundaries of $D=\Omega_{R} \backslash \bar{\Omega}_{M}$ and $\Phi(x, y)=-e^{i \beta|x-y|} /(4 \pi|x-y|)$ is the fundamental solution of the Helmholtz equation with the wave number $\beta$.

Note that for $|x|>\tilde{M}_{1}$,

$$
\int_{\Gamma_{M}} \frac{d S_{y}}{|x-y|^{2}} \leq \int_{\Gamma_{M}} \frac{d S_{y}}{(|x|-M)^{2}} \leq \frac{4 \pi M^{2}}{\left(\tilde{M}_{1}-M\right)^{2}}
$$

By Schwarz's inequality and the properties of $\Phi$,

$$
\begin{gathered}
\left|\int_{\Gamma_{M}}\left[\frac{\partial w}{\partial n}(y) \Phi(x, y)-w(y) \frac{\partial \Phi}{\partial n_{y}}(x, y)\right] d S_{y}\right|^{2} \\
\leq C e^{-2 \operatorname{Im}(\beta)|x|}\left(\left\|\frac{\partial w}{\partial n}\right\|_{L^{2}\left(\Gamma_{M}\right)}^{2}+\|w\|_{L^{2}\left(\Gamma_{M}\right)}^{2}\right) \int_{\Gamma_{M}} \frac{d S_{y}}{|x-y|^{2}} \\
\leq C e^{-2 \operatorname{Im}(\beta)|x|}\left(\|w\|_{H^{1}\left(\Omega_{1}^{c}\right)}^{2}+\|f\|_{L^{2}\left(\Omega_{1}^{c}\right)}^{2}\right) .
\end{gathered}
$$

For the last inequality above, we used an interior regularity estimate; i.e., since $w$ satisfies (6.1), its $H^{2}$-norm in a neighborhood of $\Gamma_{M}$ can be bounded by the $H^{1}$ norm of $w$ and the $L^{2}$-norm of $f$ in a slightly larger neighborhood. The analogous inequality bounding the integral on $\Gamma_{R}$ holds, and hence

$$
\begin{aligned}
\mid \int_{\Gamma_{M} \cup \Gamma_{R}}\left[\frac{\partial w}{\partial n}(y) \Phi(x, y)\right. & \left.-w(y) \frac{\partial \Phi}{\partial n_{y}}(x, y)\right]\left.d S_{y}\right|^{2} \\
& \leq C e^{-2 \operatorname{Im}(\beta)|x|}\left(\|w\|_{H^{1}\left(\Omega_{1}^{c}\right)}^{2}+\|f\|_{L^{2}\left(\Omega_{1}^{c}\right)}^{2}\right) .
\end{aligned}
$$

For the volume integral in (6.3), let $\tilde{\alpha}=\min \{\alpha, \operatorname{Im}(\beta)\}$. Then

$$
\begin{aligned}
\left|\int_{D} f(y) \Phi(x, y) d y\right| & \leq C C_{f} \int_{D} e^{-\alpha|y|} \frac{e^{-\operatorname{Im}(\beta)|x-y|}}{|x-y|} d y \\
& \leq C C_{f} e^{-\tilde{\alpha}|x|} \int_{D} \frac{1}{|x-y|} d y \\
& \leq C C_{f}|x|^{2} e^{-\tilde{\alpha}|x|} \leq C C_{f} e^{-\alpha_{1}|x|}
\end{aligned}
$$

for $|x|>\tilde{M}_{2}$ and $0<\alpha_{1}<\tilde{\alpha}$. The first inequality of Lemma 6.1 now follows from inequalities (6.4) and (6.5).

For the second inequality, let $D_{1} \subset S_{\gamma}$ be open sets such that $S_{\gamma}$ is a $\gamma$ neighborhood of $\Gamma_{\delta}$ with $\gamma$ independent of $\delta$ and $\bar{D}_{1} \subset S_{\gamma}$. Using an interior regularity estimate and integrating (6.2) over $S_{\gamma}$ gives

$$
\begin{aligned}
\|w\|_{H^{1 / 2}\left(\Gamma_{\delta}\right)} & \leq C\|w\|_{H^{2}\left(D_{1}\right)} \leq C\left(\|w\|_{L^{2}\left(S_{\gamma}\right)}+\|f\|_{L^{2}\left(S_{\gamma}\right)}\right) \\
& \leq C e^{-\alpha_{1} \delta}\left(\|w\|_{H^{1}\left(\Omega_{1}^{c}\right)}+\|f\|_{L^{2}\left(\Omega_{1}^{c}\right)}+C_{f}\right) .
\end{aligned}
$$


Proof of Proposition 3.2. We apply Lemma 6.1 with $f=0$. Since $U$ is compact it follows that $\alpha_{1}$ (in Lemma 6.1) can be chosen independent of $z \in U$. The proposition then follows from the stability of the problem (2.16).

Proof of Lemma 4.3. Let $m$ be the (algebraic) multiplicity of $\lambda$. For any non-zero $\psi \in V$,

$$
(T-\lambda I)^{m} \psi=0 .
$$

There exists a positive integer $n \leq m$, such that

$$
(T-\lambda I)^{n-1} \psi \neq 0 \quad \text { and } \quad(T-\lambda I)^{n} \psi=0 .
$$

We will show that there exist constants $\alpha, C$ and $M$ depending only on $\lambda, T$, and $n$, such that $\psi$ satisfies (4.3) with these constants. The proof is by induction on $n$. The case of $n=1$ corresponds to an eigenfunction $\psi_{1}$ and immediately follows from Lemma 6.1 since $\psi$ satisfies

$$
\Delta \psi(x)+\left(d_{0} k(\lambda)\right)^{2} \psi(x)=0 \text { for }|x|>r_{1} .
$$

Let $\psi$ satisfy (6.6) with $2 \leq n \leq m$ and denote $\psi_{j}=(T-\lambda I)^{n-j} \psi$ for $j=1, \ldots, n$. Assume that (4.3) holds for $\psi_{j}$ for $j=1, \ldots, n-1$ with constants depending only on $\lambda, T$, and $j$. We need to estimate the decay of $\psi_{n}$. Then $(T-\lambda I) \psi_{n}=\psi_{n-1}$, so outside of $\Omega_{1}$,

$$
d_{0}^{2} \psi_{n}+\lambda\left(\Delta \psi_{n}+d_{0}^{2} \psi_{n}\right)=-\left(\Delta \psi_{n-1}+d_{0}^{2} \psi_{n-1}\right) .
$$

A straightforward computation gives

$$
\Delta \psi_{n}+\left(d_{0} k(\lambda)\right)^{2} \psi_{n}=d_{0}^{2} \sum_{j=1}^{n-1} \frac{(-1)^{j+1}}{\lambda^{j+1}} \psi_{n-j} .
$$

Since the function on the right of (6.7) decays exponentially by the inductive assumption, by Lemma 6.1 there exist $\alpha=\alpha(T, \lambda, n), C=C(T, \lambda, n)$ and $M=$ $M(T, \lambda, n)$ such that $\psi_{n}$ satisfies

$$
\left|\psi_{n}(x)\right| \leq C e^{-\alpha|x|} \sum_{j=1}^{n}\left\|\psi_{j}\right\|_{H^{1}\left(\mathbb{R}^{n}\right)}
$$

for $|x|>M$. In addition, from the continuity of $T-\lambda I$ and the definition of $\psi_{j}$ there is a constant $C=C(T, \lambda, n)$ such that

$$
\left\|\psi_{j}\right\|_{H^{1}\left(\mathbb{R}^{3}\right)} \leq C\left\|\psi_{n}\right\|_{H^{1}\left(\mathbb{R}^{3}\right)},
$$

for $j=1, \ldots, n-1$. Thus, from (6.8), there exist $\alpha=\alpha(T, \lambda, n), C=C(T, \lambda, n)$, and $M=M(T, \lambda, n)$ such that

$$
\left|\psi_{n}(x)\right| \leq C e^{-\alpha|x|}\left\|\psi_{n}\right\|_{H^{1}\left(\mathbb{R}^{3}\right)}
$$

for $|x|>M$.

Proof of Lemma 4.4. We will prove a result for the truncated problem analogous to Lemma 6.1] i.e., if $\psi_{\delta} \in H_{0}^{1}\left(\Omega_{\delta}\right)$ satisfies

$$
\Delta \psi_{\delta}+\beta^{2} \psi_{\delta}=f \text { in } \Omega_{\delta} \backslash \bar{\Omega}_{1}
$$


with $\operatorname{Im}(\beta)$ positive, $f \in L^{2}\left(\Omega_{\delta} \backslash \bar{\Omega}_{1}\right)$ and there exist positive constants $\alpha, C$ and $M$ such that $|f(x)| \leq C_{f} e^{-\alpha|x|}$ for $|x|>M>r_{1}$, then there exist positive constants $\alpha_{1}, C_{1}$ and $M_{1}$ independent of $\psi_{\delta}, f$ and $\delta$ such that

$$
\left|\psi_{\delta}(x)\right| \leq C_{1} e^{-\alpha_{1}|x|}\left(\left\|\psi_{\delta}\right\|_{H^{1}\left(\Omega_{\delta} \backslash \bar{\Omega}_{1}\right)}+\|f\|_{L^{2}\left(\Omega_{\delta} \backslash \bar{\Omega}_{1}\right)}+C_{f}\right)
$$

for $|x|>M_{1}$. Once we verify (6.9), the same technique as used in the proof of Lemma 4.3 will complete the proof. Indeed, let $\tilde{V}_{\delta}$ be as in the lemma, let $\tilde{m}(i)$ be as in the discussion immediately proceeding the lemma, and set $\tilde{m}=\sum_{i} \tilde{m}(i)$. For any non-zero $\psi \in \tilde{V}_{\delta}$,

$$
\prod_{i=1}^{k}\left(T_{\delta}-\lambda_{i}^{\delta} I\right)^{\tilde{m}(i)} \psi=\prod_{i=1}^{\tilde{m}}\left(T_{\delta}-\tilde{\lambda}_{i} I\right) \psi=0,
$$

with the obvious definition of $\tilde{\lambda}_{i}$. There is a positive integer $n \leq \tilde{m}$ such that

$$
\prod_{i=1}^{n-1}\left(T_{\delta}-\tilde{\lambda}_{i} I\right) \psi \neq 0 \text { and } \prod_{i=1}^{n}\left(T_{\delta}-\tilde{\lambda}_{i} I\right) \psi=0 .
$$

Setting $\psi_{n}=\psi$ and $\psi_{j}=\left(T_{\delta}-\tilde{\lambda}_{n-j} I\right) \psi_{j+1}$ for $j=1, \ldots, n-1$, we have

$$
\Delta \psi_{n}+\left(d_{0} k\left(\lambda_{1}\right)\right)^{2} \psi_{n}=d_{0}^{2} \sum_{j=1}^{n-1} \frac{(-1)^{j+1}}{\Pi_{l=1}^{j+1} \tilde{\lambda}_{l}} \psi_{n-j} \text { in } \Omega_{\delta} \backslash \Omega_{1}^{c} .
$$

Recall that the norm of $T_{\delta}$ is bounded by a constant independent of $\delta$ from (3.1) and (3.2) and that $\tilde{\lambda}_{i}$, for each $i$, is inside $\Gamma$, so that $\operatorname{Im}\left(d_{0} k\left(\tilde{\lambda}_{i}\right)\right)>0$. Thus we can apply the induction on $n$ to (4.4) as in the proof of Lemma 4.3.

We now verify (6.9). We start by decomposing $\psi_{\delta}=\psi+w$, where $\psi$ is defined to be equal to $\psi_{\delta}$ in $\Omega_{1}$ and satisfies

$$
\begin{aligned}
\Delta \psi+\beta^{2} \psi & =\tilde{f} \text { in } \Omega_{1}^{c}, \\
\psi & =\psi_{\delta} \text { on } \Gamma_{1},
\end{aligned}
$$

where $\tilde{f}$ is the zero extension of $f$ to $\Omega_{\delta}^{c}$. Then $w$ satisfies the equations

$$
\begin{aligned}
\Delta w+\beta^{2} w & =0 \quad \text { in } \Omega_{\delta} \backslash \bar{\Omega}_{1}, \\
w & =0 \text { on } \Gamma_{1}, \\
w & =-\psi \text { on } \Gamma_{\delta} .
\end{aligned}
$$

Note that $\psi$ decays exponentially by Lemma 6.1 and the stability of (6.10) implies

$$
|\psi(x)| \leq C_{1} e^{-\alpha_{1}|x|}\left(\left\|\psi_{\delta}\right\|_{H^{1}\left(\Omega_{\delta} \backslash \bar{\Omega}_{1}\right)}+\|f\|_{L^{2}\left(\Omega_{\delta} \backslash \bar{\Omega}_{1}\right)}+C_{f}\right)
$$

for $|x|>M_{1}$. So we have only to show exponential decay of $w$.

We do this by showing that

$$
\|w\|_{H^{2}\left(\Omega_{\delta} \backslash \bar{\Omega}_{1}\right)} \leq C_{\delta}\|\psi\|_{H^{2}\left(S_{\epsilon} \cap \Omega_{\delta}\right)}
$$

where $S_{\epsilon}$ is an $\epsilon$-neighborhood of $\Gamma_{\delta}$ for $\epsilon>0$. Here $C_{\delta}$ only grows as a polynomial of $\delta$. Using (6.12) gives (for $\epsilon^{\prime}>\epsilon$ independent of $\delta$ )

$$
\begin{aligned}
\|w\|_{H^{2}\left(\Omega_{\delta} \backslash \bar{\Omega}_{1}\right)} & \leq C_{\delta}\|\psi\|_{L^{2}\left(S_{\epsilon^{\prime}}\right)} \\
& \leq C_{\delta} e^{-\alpha_{1} \delta}\left(\|\psi\|_{H^{1}\left(\Omega_{1}^{c}\right)}+\|f\|_{L^{2}\left(\Omega_{\delta} \backslash \bar{\Omega}_{1}\right)}+C_{f}\right) \\
& \leq C_{1} e^{-\alpha_{2}|x|}\left(\left\|\psi_{\delta}\right\|_{H^{1}\left(\Omega_{\delta} \backslash \bar{\Omega}_{1}\right)}+\|f\|_{L^{2}\left(\Omega_{\delta} \backslash \bar{\Omega}_{1}\right)}+C_{f}\right) .
\end{aligned}
$$


Here we absorbed the polynomial growth in $C_{\delta}$ by making $\alpha_{2}<\alpha_{1}$. Combining the above inequalities with a Sobolev imbedding theorem proves (6.9.).

Finally, to prove (6.12), we decompose $w=\tilde{w}+w_{0}$, where $\tilde{w}=-\chi \psi$ and $\chi$ is a cutoff function which is defined on $\Omega_{\delta} \backslash \bar{\Omega}_{1}$, is one in a neighborhood of $\Gamma_{\delta}$ and vanishes outside of $S_{\epsilon} \cap\left(\Omega_{\delta} \backslash \bar{\Omega}_{1}\right)$. We need only show that

$$
\left\|w_{0}\right\|_{H^{2}\left(\Omega_{\delta} \backslash \bar{\Omega}_{1}\right)} \leq C_{\delta}\|\psi\|_{H^{2}\left(S_{\epsilon} \cap \Omega_{\delta}\right)} .
$$

Note that $w_{0}$ satisfies

$$
\begin{aligned}
\Delta w_{0}+\beta^{2} w_{0} & =g \text { in } \Omega_{\delta} \backslash \bar{\Omega}_{1}, \\
w_{0} & =0 \text { on } \Gamma_{1} \cup \Gamma_{\delta},
\end{aligned}
$$

where $g=-\left(\Delta \tilde{w}+\beta^{2} \tilde{w}\right)$ is in $L^{2}\left(S_{\epsilon} \cap\left(\Omega_{\delta} \backslash \bar{\Omega}_{1}\right)\right)$. Clearly, $\left\|w_{0}\right\|_{H^{1}\left(\Omega_{\delta} \backslash \bar{\Omega}_{1}\right)}$ is bounded by $C\|g\|_{L^{2}\left(\Omega_{\delta} \backslash \bar{\Omega}_{1}\right)}$.

Let $\Omega_{2}$ be a ball centered at the origin and of radius $r_{2}>r_{1}$, independent of $\delta$, and contained in $\Omega_{\delta}$. Let $\chi_{1}$ be a cutoff function on $\Omega_{\delta} \backslash \bar{\Omega}_{1}$ which is one on $\Omega_{\delta} \backslash \bar{\Omega}_{2}$ and vanishes near $\Gamma_{1}$. Then $\left(1-\chi_{1}\right) w_{0}$ and $\chi_{1} w_{0}$ (extended by zero in $\Omega_{1}$ ) satisfy equations similar to (6.14) on domains $\Omega_{2} \backslash \bar{\Omega}_{1}$ and $\Omega_{\delta}$, respectively. The data for these problems involves $g$ above and at most first order derivatives of $w_{0}$, and hence is controlled in $L^{2}\left(\Omega_{\delta} \backslash \bar{\Omega}_{1}\right)$. It follows from a regularity on the smooth domain $\Omega_{2} \backslash \bar{\Omega}_{1}$ that

$\left\|(1-\chi) w_{0}\right\|_{H^{2}\left(\Omega_{\delta} \backslash \bar{\Omega}_{1}\right)}=\left\|(1-\chi) w_{0}\right\|_{H^{2}\left(\Omega_{2} \backslash \bar{\Omega}_{1}\right)} \leq C\left(\|g\|_{L^{2}\left(\Omega_{2} \backslash \bar{\Omega}_{1}\right)}+\left\|w_{0}\right\|_{H^{1}\left(\Omega_{2} \backslash \bar{\Omega}_{1}\right)}\right)$.

Finally, by dilation to a fixed sized domain,

$$
\left\|\chi w_{0}\right\|_{H^{2}\left(\Omega_{\delta} \backslash \bar{\Omega}_{1}\right)}=\left\|\chi w_{0}\right\|_{H^{2}\left(\Omega_{\delta}\right)} \leq C_{\delta}\left(\|g\|_{L^{2}\left(\Omega_{\delta} \backslash \bar{\Omega}_{1}\right)}+\left\|w_{0}\right\|_{H^{1}\left(\Omega_{\delta} \backslash \bar{\Omega}_{1}\right)}\right) .
$$

The inequality (6.12) follows by combining the above.

Proof of Lemma 4.5. The $H^{1}$-estimate for $\left(T-T_{\delta}\right) u$ will be computed in two subdomains $\Omega_{\delta}$ and $\Omega_{\delta}^{c}$. First, note that since $T u$ is the solution to the problem

$$
A_{1}(T u, \phi)=B(u, \phi) \text { for } \phi \in H^{1}\left(\mathbb{R}^{3}\right)
$$

it satisfies

$$
\Delta T u+d_{0}^{2} T u=-d_{0}^{2} u \text { in } \Omega_{1}^{c} .
$$

It follows from Lemma 6.1 that

$$
\|T u\|_{H^{1 / 2}\left(\Gamma_{\delta}\right)} \leq C_{1} e^{-\alpha_{1} \delta}\left(\|T u\|_{H^{1}\left(\Omega_{1}^{c}\right)}+\|u\|_{H^{1}\left(\Omega_{1}^{c}\right)}\right)
$$

for $\delta>M_{1}$.

Take $M_{1}>\delta_{0}$ in Theorem 3.1. In $\Omega_{\delta}, \psi \equiv\left(T-T_{\delta}\right) u$ is the unique solution to the problem

$$
\begin{aligned}
A_{1}(\psi, \phi) & =0 \text { for all } \phi \in H_{0}^{1}\left(\Omega_{\delta}\right), \\
\psi & =T u \text { on } \Gamma_{\delta},
\end{aligned}
$$

so that by stability and (6.16),

$$
\begin{aligned}
\left\|\left(T-T_{\delta}\right) u\right\|_{H^{1}\left(\Omega_{\delta}\right)} & \leq C\|T u\|_{H^{1 / 2}\left(\Gamma_{\delta}\right)} \\
& \leq C_{1} e^{-\alpha_{1} \delta}\left(\|T u\|_{H^{1}\left(\mathbb{R}^{3}\right)}+\|u\|_{H^{1}\left(\mathbb{R}^{3}\right)}\right)
\end{aligned}
$$

for $\delta>M_{1}$.

In $\Omega_{\delta}^{c}, \psi \equiv\left(T-T_{\delta}\right) u=T u$ is the unique solution to (6.15) with the boundary condition $T u$ on $\Gamma_{\delta}$. By stability,

$$
\|T u\|_{H^{1}\left(\Omega_{\delta}^{c}\right)} \leq C\left(\|u\|_{L^{2}\left(\Omega_{\delta}^{c}\right)}+\|T u\|_{H^{1 / 2}\left(\Gamma_{\delta}\right)}\right) .
$$


Integrating the square of (4.5) over $\Omega_{\delta}^{c}$ and using (6.16) gives

$$
\|T u\|_{H^{1}\left(\Omega_{\delta}^{c}\right)} \leq C_{\delta} e^{-\alpha_{1} \delta}\left(\|u\|_{H^{1}\left(\mathbb{R}^{3}\right)}+\|T u\|_{H^{1}\left(\mathbb{R}^{3}\right)}\right)
$$

for $\delta>M_{1}$ and $C_{\delta}$ a linear function of $\delta$. The $\delta$-dependence in the constant can be removed by making $\alpha_{1}$ slightly smaller. The result follows from (6.17), (6.18) and the boundedness of $T$.

\section{REFERENCES}

[1] J. Aguilar and J. M. Combes. A class of analytic perturbations for one-body Schrödinger Hamiltonian. Commun. Math. Phys., 22:269-279, 1971. MR0345551 (49:10287)

[2] S. Balay, K. Buschelman, V. Eijkhout, W. D. Gropp, D. Kaushik, M. G. Knepley, L. C. McInnes, B. F. Smith, and H. Zhang. PETSc users manual. Technical Report ANL95/11 - Revision 2.1.5, Argonne National Laboratory, 2004.

[3] E. Balslev and J. M. Combes. Spectral properties of many body Schrödinger operators with dilation analytic interactions. Commun. Math. Phys., 22:280-294, 1971. MR.0345552 (49:10288)

[4] J.-P. Bérenger. A perfectly matched layer for the absorption of electromagnetic waves. J. Comput. Phys., 114(2):185-200, 1994. MR1294924 (95e:78002)

[5] J.-P. Bérenger. Three-dimensional perfectly matched layer for the absorption of electromagnetic waves. J. Comput. Phys., 127(2):363-379, 1996. MR.1412240(97h:78001)

[6] D. Boffi. Fortin operator and discrete compactness for edge elements. Numer. Math., 87(2):229-246, 2000. MR1804657 (2001k:65168)

[7] D. Boffi, P. Fernandes, L. Gastaldi, and I. Perugia. Computational models of electromagnetic resonators: analysis of edge element approximation. SIAM J. Numer. Anal., 36(4):1264-1290 (electronic), 1999. MR1701792 (2000g:65112)

[8] J. H. Bramble and J. E. Osborn. Rate of convergence estimates for nonselfadjoint eigenvalue approximations. Math. Comp., 27:525-549, 1973. MR0366029 (51:2280)

[9] J. H. Bramble and J. E. Pasciak. Analysis of a finite PML approximation for the three dimensional time-harmonic Maxwell and acoustic scattering problems. Math. Comp., 76:597614, 2007. MR2291829 (2008b:65130)

[10] W. Chew and W. Weedon. A 3D perfectly matched medium for modified Maxwell's equations with stretched coordinates. Microwave Opt. Techno. Lett., 13(7):599-604, 1994.

[11] F. Collino and P. Monk. The perfectly matched layer in curvilinear coordinates. SIAM J. Sci. Comput., 19(6):2061-2090, 1998. MR1638033 (99e:78029)

[12] D. Colton and R. Kress. Inverse Acoustic and Electromagnetic Scattering Theory. SpringerVerlag, New York, 1998. MR1635980 (99c:35181)

[13] S. Hein, T. Hohage, and W. Koch. On resonances in open systems. J. Fluid Mech., 506:225284, 2004. MR2259489(2007d:76204)

[14] S. Hein, T. Hohage, W. Koch, and J. Schöberl. Acoustic resonances in a high lift configuration. J. Fluid Mech., 582:179-202, 2007. MR2331498

[15] V. Hernandez, J. E. Roman, and V. Vidal. SLEPc: A scalable and flexible toolkit for the solution of eigenvalue problems. ACM Transactions on Mathematical Software, 31(3):351362, Sept. 2005. MR2266798

[16] P. D. Hislop and I. M. Sigal. Introduction to Spectral Theory with Applications to Schrödinger Operators. Springer, Berlin-Heidelberg-New York, 1996. MR.1361167 (98h:47003)

[17] T. Kato. Perturbation Theory for Linear Operators. Springer-Verlag, Berlin-Heidelberg-New York, 1976. MR0407617 (53:11389)

[18] S. Kim and J. E. Pasciak. Analysis of a Cartesian PML approximation to acoustic scattering problems in $\mathbb{R}^{2}$. In preparation.

[19] F. Kukuchi. On a discrete compactness property for the Nédélec finite elements. J. Fac. Sci. Univ. Tokyo, Sect. 1A, Math, 36:479-490, 1989. MR1039483 (91h:65173)

[20] M. Lassas and E. Somersalo. Analysis of the PML equations in general convex geometry. Proc. Roy. Soc. Edinburgh Sect. A, 131(5):1183-1207, 2001. MR.1862449 (2002k:35020)

[21] P. Monk. Finite Element Methods for Maxwell's Equations. Numerical Mathematics and Scientific Computation. Oxford Univeristy Press, Oxford, UK, 2003. MR2059447|(2005d:65003) 
[22] P. Monk and L. Demkowicz. Discrete compactness and the approximation of Maxwell's equations in $\mathbb{R}^{3}$. Math. Comp., 70(234):507-523, 2001. MR.1709155 (2001g:65156)

[23] J. E. Osborn. A note on a perturbation theorem for the matrix eigenvalue problem. Numer. Math., 13:152-153, 1969. MR0246502 (39:7806)

[24] B. Simon. The theory of resonances for dilation analytic potentials and the foundations of time dependent perturbation theory. Ann. Math., 97:247-274, 1973. MR0353896 (50:6378)

[25] L. N. Trefethen. Pseudospectra of linear operators. SIAM Rev., 39(3):383-406, 1997. MR.1469941 (98i:47004)

[26] M. Zworski. Numerical linear algebra and solvability of partial differential equations. Commun. Math. Phys., 229:293-307, 2002. MR1923176 (2003i:35008)

Department of Mathematics, Texas A\&M University, College Station, Texas 778433368

E-mail address: sgkim@math.tamu.edu

Department of Mathematics, Texas A\&M University, College Station, Texas 778433368

E-mail address: pasciak@math.tamu.edu 